\title{
Supporting Sustainability Decisions in Large Organisations
}

\author{
David Stefan \\ Urban Sustainability \& Resilience Centre \\ University College London \\ London, United Kingdom \\ d.stefan@ucl.ac.uk
}

\author{
Emmanuel Letier \\ Department of Computer Science \\ University College London \\ London, United Kingdom \\ e.letier@ucl.ac.uk
}

\begin{abstract}
Sustainability management systems are important technologies used by large organisations to monitor and control their $\mathrm{CO} 2$ emissions and other environmental impacts. So far, these systems have been designed to support data collection and reporting mandated by regulations, but they provide little support for decision making in order to improve an organisation's environmental sustainability. In this paper, we present a systematic method to help organisations make such decisions. Our method builds on goal-oriented requirements engineering for modelling an organisation's sustainability goals and on statistical decision analysis for guiding decisions under uncertainty. By being explicit about uncertainty, our method provides a sound assessment of the risks associated with alternative options (including the "business as usual" option), and, through the concept of information value, informs decision makers whether reducing uncertainty through additional data collection will be useful for their decisions. We illustrate our approach on real sustainability decision questions at UCL's Department of Computer Science.

Index Terms - Sustainability management, requirements engineering, goal modelling, decision analysis, uncertainty, information value.
\end{abstract}

\section{INTRODUCTION}

Climate change and its accompanying sustainability concerns are widely considered to be the defining themes of the $21^{\text {st }}$ century. Leading climate scientists and economic analysts have repeatedly established the severity of the issue and the scale of the challenge [1], [2]. Large organisations play an important role in helping to mitigate and adapt to the consequences of climate change - as recent World Resource Institute report suggested, the engagement of the private sector is vital to ensure widespread support for sustainability and climate action [3].

But making sustainability decisions at large organisations is hard. It involves trade-offs between multiple long- and shortterm objectives that must be made under strong budgetary constraints, uncertainties about the future evolution of many system variables, and sometimes simply the lack of shared understanding of what the real objectives and the potential impacts of various decisions on these objectives are [4]-[6].

Today, large organisations use sustainability management information systems to monitor and control their $\mathrm{CO}_{2}$ emissions and environmental impacts. The design of the current generation of these systems is however largely data-centric - it concentrates on data collection and reporting, mostly to satisfy new regulations concerning the reporting and trading of carbon emissions. Deploying these systems allows organisations to make significant reductions in energy use and carbon emissions in the first few years, but beyond mandatory reporting, it is difficult to identify the most effective actions that would efficiently address the risks associated with wider sustainability issues [7].

One limitation of these systems is that little attention has been given to the information needs of the decision makers when making sustainability-related decisions [8]. This leads to thwarting value generation opportunities, as the right information is not available to the right people at the right time [9]. A recent Ernst $\&$ Young report on corporate sustainability concluded that inefficient environmental management software systems pose challenges to find the right data and assess its credibility and relevance [10].

Another limitation concerns the lack of support for reasoning about uncertainty, assessing the risks associated with uncertainty, and assessing the cost and value of reducing uncertainty through additional data collection and analysis where possible. Uncertainty is the lack of complete knowledge about the actual value of some quantity of interest, for example, the current number of computers in the organisation or the price of energy in 5 years' time. Ignoring uncertainty may expose an organisation to significant risk of making wrong decisions and not achieving desired strategic goals. Most policy decisions are however founded on deterministic analysis that ignore uncertainty [11].

Our objective is to address these limitations by presenting a systematic method for making sustainability decisions under uncertainty where the decision makers' information needs are derived from the organisation's sustainability goals, the decisions to be made, and the organisation's current uncertainty about the quantities of interests to these decisions. Our method builds on goal-oriented requirements engineering techniques [12]-[14] to elicit and model an organisation's sustainability goals, and on statistical decision analysis [15], [16] to support multi-objective decisions under uncertainty.

We illustrate our method on a case study of sustainability decisions at UCL Department of Computer Science in which we assess two projects for their impact on UCL strategic sustainability goals, analyse their financial implications, and identify 
where reducing uncertainty about decision parameters is most profitable for the organisation.

\section{MOtIVATING EXAMPLE}

University College London (UCL) is a large university with its main campus located in the centre of London. Operating UCL resulted in 61,000 tonnes of CO2 in the 2011/12 academic year, contributed mainly by the university's energy consumption related to heating, computing and construction activities [17]. The target for reducing carbon dioxide emissions was set to $34 \%$ of the $2005 / 06$ baseline $\left(64,000 \mathrm{t} \mathrm{CO}_{2}\right)$ by year 2020 . With a steady growth in the number of students and staff [18], expanding campus and tightening legislation, the university must implement measures to reduce its energy consumption in order to achieve its $\mathrm{CO}_{2}$ reduction targets.

UCL's Computer Science department (CS) is research oriented with a heavy emphasis on experimentation. It operates on eight floors in three different buildings, employs around 600 members of staff and yearly enrols more than 500 students. In 2013, the department consumed $9 \mathrm{GWh}$ of energy and produced an equivalent of 6,000 tonnes of $\mathrm{CO}_{2}$ emissions. More than a third of this energy consumption (i.e. around $3 \mathrm{GWh}$ ) is related to computing. This is mainly due to a $130 \mathrm{~m}^{2}$ data centre and around 600 desktop computers used for teaching, research and administrative purposes.

In the same year, the CS Technical Support Group considered two projects that would reduce the department's energy use and $\mathrm{CO}_{2}$ emissions. The first, the Thin Clients (TC) project, would consist in replacing 100 desktop computers with low powered thin clients. The second, the Data Centre Migration (DCM) project, would consist in partially migrating the department's large data centre to an external data centre provider with a more efficient energy use. The Technical Support Group later decided to fund the TC project but rejected the DCM project due to data security concerns and other legal issues related to external management of sensitive data.

Regardless of what decision has been made, we will use the information about the two projects in retrospect to demonstrate the individual steps of our method. Different groups throughout UCL have similar sustainability-related project ideas but have difficulties assessing the impacts of their projects on the university's sustainability goals. There is thus a real need for management tools to support these groups in evaluating the sustainability impacts of their projects and to support the organisation as a whole to choose the most effective alternatives.

\section{RELATED WORK}

Software systems supporting the adoption of sustainability strategies have evolved as a response to growing regulatory pressure from governments and non-governmental organisations. These systems are regarded in the literature mostly as environmental enterprise resource planning (ERP) software [7], [8] that primarily aim at collecting large volume of data and show compliance with regulations. Examples of the most popular software include Hara, Credit360 or CA ecoSoftware [5]. These software systems are particularly beneficial for corporations with a large customer base and presence in various countries with different legislation. They remain rudimentary, however, especially when compared with other tools used for reporting financial measures [10] and provide very little decision support.

Others have previously applied goal-oriented requirements engineering techniques to sustainability decisions: Cabot et al. and Mahaux et al. have both applied goal modelling techniques to the organisation of large events [19], [20]; and Penzenstadler and Femmer proposed generic goal-modelling framework that supports the assessment of environmental sustainability impact of software engineering projects [21]. However, all take a qualitative approach where sustainability goals remain vague and immeasurable. These methods help to identify the main sustainability concerns and the forces affecting them, but they provide limited support for complex decision-making. By contrast, we use a quantitative approach where goals are given formal, measurable definitions [22]. Our models include multiple, organisation-specific sustainability goals defined in terms of domainspecific variables that are related to each other through refinement equations [13]. Such models allow one to reason quantitatively about the impacts of alternative options on the levels of goal attainment and make trade-offs among them.

The Inflo tool provides support for collaborative elaboration and management of quantitative decision models, notably for supporting sustainability decisions [23], [24]. Using Inflo, one can, for example, develop an argument to compare the carbon emissions resulting from printing a text on a paper versus reading the same text on a computer screen. Inflo provides support for model elaboration and execution, but not for decisions under uncertainty. Inflo models could be used as an input for the model elaboration step of our method.

\section{SUSTAINABILITY DECISIONS UNDER UNCERTAINTY}

Our approach to the problem of making sustainability decisions in large organisations is structured into four steps:

1. Elaborating sustainability decision models

2. Eliciting model parameter values

3. Analysing costs, benefits, and risks

4. Analysing information value

These steps adapt existing goal modelling [12] and statistical decision analysis techniques [15] to the particular context of sustainability decisions in large organisation. Our approach has been strongly influenced by our recent work on software requirements and architecture decisions under uncertainty [25].

The first step consists in elaborating a strategic sustainability goal model for the whole organisation, and a series of concernspecific impact models relating detailed, low-level variables (such as the number and type of computers used in the organisation) to variables of interest to the decision makers in the strategic sustainability goal model. The second step consists in eliciting the model parameters' probability distributions that characterise uncertainty about their values. The third step involves computing the expected costs, benefits and risks of alternative choices through Monte-Carlo simulation. The fourth step consists in measuring the expected value of perfect information [26] 


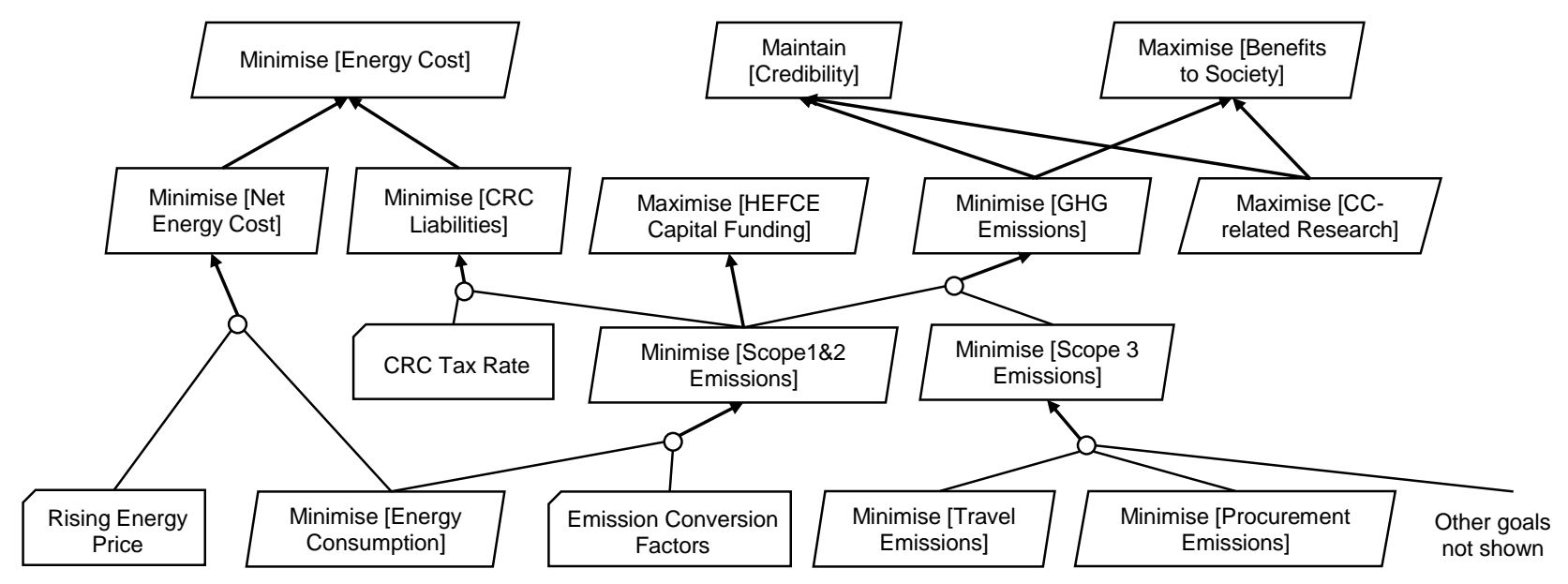

Fig. 1. A portion of UCL sustainability strategic goal model (adapted from [27]).

for the decision problem. Measuring the expected value of perfect information allows decision makers to assess whether it would be profitable to reduce some of the decision problem uncertainty before committing to a particular choice, and it guides them in identifying which uncertainty to reduce.

The following sections present each of these steps in turn and illustrate their applications on our motivating example.

\section{Elaborating Sustainability Decisions Models}

The first step consists in elaborating sustainability decision models. We distinguish models at two different levels of abstraction: strategic sustainability goal model defines the overall sustainability goals of the whole organisation and provides reference criteria against which to evaluate individual projects; concern-specific impact models are partial models describing the impacts of a large number of low-level variables (such as the number of devices of various types, their usage patterns, their energy consumption, etc.) on leaf goals in the strategic sustainability goal model (e.g. overall energy consumption). In other words, the strategic sustainability goal model defines the "big picture", and the concern-specific impact models allow one to relate details from various parts of an organisation to this big picture.

\section{A. Defining the organisation's strategic sustainability goals}

We rely on an existing goal-oriented requirements engineering method [12]-[14] called KAOS (Knowledge Acquisition in Automated Specification) to elicit and elaborate the strategic sustainability goal model. In the KAOS goal modelling approach, goals are prescriptive statements of intent to be satisfied through the cooperation of agents. Agents in this context refer to software systems, hardware devices and humans. Goals are defined precisely in terms of domain-specific phenomena. Levels of goals satisfaction can be defined through measurable objective functions defined in terms of quality variables attached to each goal. A typical example of environmental sustainability goal for an organisation would be to minimise its greenhouse gas emissions.
Goals can be refined into subgoals and domain assumptions. Domain assumptions are statements about the application domain that are outside the control of the organisation, for example statements concerning energy cost, or the amount of greenhouse gas emission per energy consumption for a specific external source of energy. In goal refinement links, refinement equations are defined to relate quality variables of a parent goal to quality variables of its subgoals and variables in the related domain assumptions.

In our application of goal modelling to sustainability decisions in large organisation, we have not defined a clear-cut criterion for deciding when to stop refining goals into subgoals. The refinement of strategic goals should proceed until when further refinements would lead to goals referring to variables that are too detailed and outside the immediate interests of strategic decision makers.

Case study. Figure 1 shows a portion of the strategic sustainability goal model at UCL [27]. We have elaborated this model from information contained in the last UCL carbon management plan and sustainability strategy [17], [28], from information elicited during interviews with sustainability programme managers and general data about UCL, energy prices, and greenhouse gas emission conversion factors.

Two important objectives are to minimize energy cost and greenhouse gas (GHG) emissions. The goal to minimize GHG emissions is further refined into two subgoals based on the definition of emissions' scope by the Greenhouse Gas Protocol. Carbon emissions of scope 1 and 2 are produced either directly on the campus (e.g. by energy production of the on-site CHP plant), or result from an external production of energy used for the operation of the university. Scope 3 emissions are all other indirect emissions related to activities of the institution such as travel, procurement, water usage, waste disposal, and energy consumed in leased buildings and facilities [29].

UCL's objective to reduce its carbon emissions by $34 \%$ by 2020 only concerns emissions of scope 1 and 2 . The reduction target was set by the Higher Education Funding Council for England (HEFCE) as a condition for universities to keep their full 


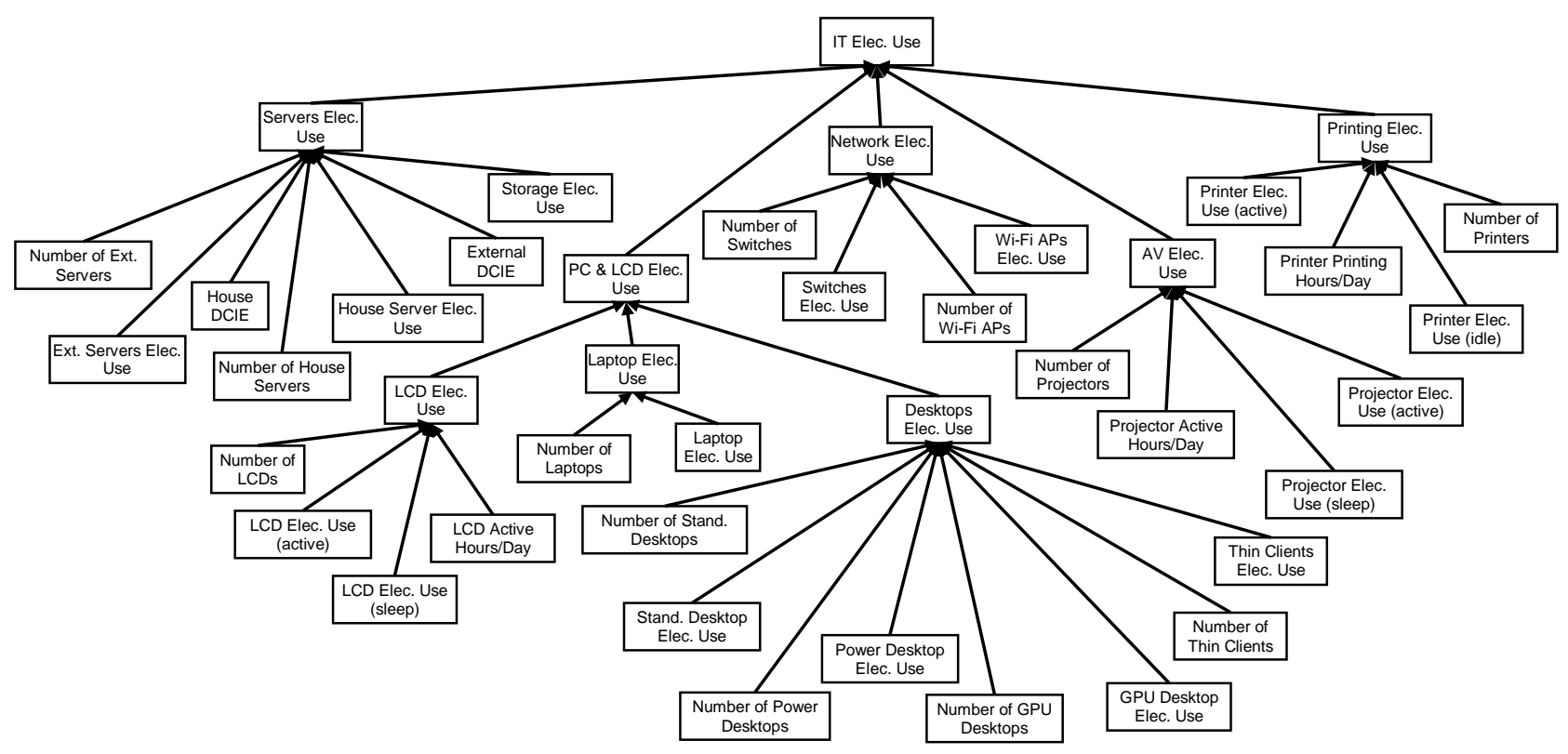

Fig. 2. Graphical representation of the ICT energy consumption impact model. Arrows assign child-nodes to their parent-nodes and appoint which lower-level variables are involved in the definition of higher-level variables.

level of capital funding, and is related to the Government's general targets of reducing UK carbon emissions by $80 \%$ by 2050 against the 1990 levels [30]. Achieving this goal will also reduce UCL's financial liability to purchase carbon emission allowances under the CRC Energy Efficiency Scheme (CRC) which is a mandatory scheme aimed at improving energy efficiency and reducing emissions in large public and private sector organisations in the UK [31]. The generic goal of reducing GHG emissions will contribute to the goal of maintaining UCL's credibility as a world-leading research and teaching institution, notably in areas related to energy, climate change, and environmental sustainability. It will also help to maximize UCL's benefits to society by contributing to the effort of mitigating and adapting to the consequences of climate change.

For the CS Thin Client and Datacentre Migration projects, we will focus on two goals: Minimise [Energy Costs] and Minimise [Scope 1\&2 Emissions]. In our model, the goal Minimise [Energy Cost] is defined in terms of a quality variable

$$
\text { EnrgCost: Year } \rightarrow N
$$

that denotes the total energy cost per year. The value of this variable is defined in terms of the refinement equation

$$
\text { EnrgCost }=\text { NetEnrgCost }+ \text { CRCLiabilities }
$$

which refers to the variables attached to the subgoals Minimise [Net Energy Cost] and Minimise [CRC Liabilities], respectively. The net energy cost is itself computed from the following equation and the CRC Liabilities computed from

$$
\text { CRCLiabilities }=\text { Scope1\&2_Emissions } * \text { CRCTaxRate. }
$$

The full quantitative model with all refinement equations is available at http://davidstefan.org/ICT4S.

Our strategic sustainability goal model has no quantitative definition and refinement equations for the goals Maintain [UCL Credibility] and Maximise [UCL Benefits to Society]. Defining meaningful, measurable quality variables for these goals is possible [16] but requires further work. When evaluating the Thin Client and the Data Centre Migration projects, we will assess their impacts on minimising UCL scope 1 and 2 emissions which contribute to an unknown degree to the goals of maximising UCL reputation and positive impact on society.

\section{B. Defining Concern-Specific Impact Models}

The concern-specific impact models define equations relating detailed variables directly affected by some project to quality variables in the strategic sustainability goal model. These models allow projects' champions to assess and communicate the impact of their projects on the overall goals of the organisation. In order to avoid developing and validating these models from scratch, it would be useful for an organisation to curate a set of concern-specific impact models that could be reused across the analyses of different projects. The concerns addressed by different models would include, for example, the impact of ICT on energy consumption, the impact of ICT on waste, the impact of heating on energy consumption, etc. A collaborative web-based modelling tool such as Inflo [23] would be extremely useful to develop, validate, manage, and reuse such models. 
These impact models are not goal models in the sense that the term is used in requirements engineering because the variables involved in these models, for example the number of thin clients used in the organisation, are not easily attached to goals.

Case study. To compute the impacts of the Thin Client and the Data Centre Migration projects, we have use a standard model for estimating energy consumption of ICT estates in UK higher education institutions [32]. This model, which is implemented as a spreadsheet, was originally developed by the UK government to help higher education institutions estimate the environmental impact of their ICT estate and is now being used by governmental departments and agencies to report their operational energy consumption footprints. Figure 2 shows a graphical representation of the variables involved in this model and the relationships among them.

\section{ELICITING MODEL PARAMETER VALUES}

The next step consists in eliciting the model parameter values for quantities that are known with certainty or their probability distributions for quantities that are uncertain. Simple, effective methods exists for eliciting such probability distributions [33]; for example, one common technique consists in eliciting a $90 \%$ confidence interval for a parameter's value, i.e. the range of values that the estimators believe has a $90 \%$ chance of containing the true value [16]. Our method is however not constrained to the use of any particular elicitation technique - one could use normal distributions and well as uniform or triangular distributions to represent uncertainty about parameter values.

Case Study. We have estimated the model parameter values of our strategic sustainability goal model, such as the current and projected future value for energy prices, CRC tax rate, and emission conversion factors from publicly available data and analyses [31], [34]. For the model parameters of the ICT-energy impact model shown in Figure 2, we have elicited values from the Head of Technical Support Group at CS. Some of these values are shown in Table I and II. When a parameter is known with certainty, the table shows a single value; when there is uncertainty, the table shows the lower and upper bounds of the $90 \%$ confidence interval.

TABLE I. Examples of a few parameter values for the ICT estate at UCL's Computer Science Department.

\begin{tabular}{lc}
\hline \multicolumn{1}{c}{ parameter } & value \\
\hline Number of House Servers & 650 \\
House Server Elec. Use & {$[100,250] \mathrm{W}$} \\
House DCIM & {$[2.1,2.6]$} \\
Number of Stand. Desktops & {$[180,220]$} \\
Stand. Desktops Elec. Use & {$[200,300]$} \\
Number of LCDs & {$[500,600]$} \\
LCD Elec. Use Active & {$[50,80] \mathrm{W}$} \\
\hline
\end{tabular}

\section{ANALYSING COSTS, BENEFITS, AND RISKS}

The next step consist in analysing the costs, benefits and risks associated with alternative decisions which can include the decision to implement either one of the proposed projects, a combination of the projects, but also the decision not to implement any of them (the "business as usual" option).

Costs may include expenditures related to the purchase of new capital, construction activities and other costs associated with projects implementation.

Benefits are related to the satisfaction of strategic goals. This can be goals related directly to stakeholders' financial interest, such as the savings resulting from reducing energy consumption, or other strategic goals, for example reducing $\mathrm{CO}_{2}$ emissions or improving reputation.

Risks are associated with the possibility of not achieving desired goals, or satisfying goals below a desired level.

\section{A. Computing projects' impacts on goals' attainment}

The computation is accomplished by evaluating the strategic goal model for inputs obtained from evaluating the concern-specific impact models for parameter values elicited for individual projects.

It is generally not possible to evaluate the models analytically because the probability distributions of the input variables and the refinement equations can be arbitrarily complex. We therefore use Monte Carlo (MC) simulation which computes the outputs as sample probability distributions by evaluating the model for a large number of random draws of values from the input probability distributions. The result of a MC simulation is a vector where each element corresponds to one evaluation of the models associated to a single such draw.

Once the model has been evaluated, we proceed with the analysis of the resulting levels of goal attainment. Since the levels of goal attainment are computed as sample probability distributions, we can infer statistical measures such as the expected level of goal attainment, or the probability that the level of goal attainment is below (or above) a desired target.

Case study. The CS Technical Support Group is considering four alternatives. The "business as usual" scenario involves the replacement of 100 desktop computers that are approaching the end of their lifetime with desktops that have similar energy use profile.

DR Replace old desktops with newer machines that have similar energy use profile.

TC Replace old desktops with thin clients that use less energy.

TABLE II. Examples of the changes in ICT parameters values after migrating 150 servers to an external datacentre.

\begin{tabular}{cccc}
\multicolumn{4}{c}{ Datacentre migration } \\
\hline parameter & current & change & new \\
\hline In-house servers number & 650 & -150 & 500 \\
External servers number & 0 & +150 & 150 \\
\hline
\end{tabular}


TABLE III. Impacts of decision alternatives on key strategic goals.

\begin{tabular}{lrrrr}
\hline & \multicolumn{1}{c}{$D R$} & \multicolumn{1}{c}{$T C$} & \multicolumn{1}{c}{$D R+D C M$} & \multicolumn{1}{c}{$T C+D C M$} \\
\hline Expected 5y Energy Cost & $£ 2,238,966$ & $£ 2,203,077$ & $£ 2,040,483$ & $£ 2,004,594$ \\
Expected 5y Project Cost & $£ 35,002$ & $£ 29,983$ & $£ 215,126$ & $£ 210,108$ \\
Expected Scope 1\&2 Emissions & 1,706 & 1,677 & 1,554 & 1,526 \\
$P($ IT Scp1\&2 reduced by 5\%) & $0 \%$ & $\sim 0 \%$ & $79 \%$ & $88 \%$ \\
$P($ Scp1\&2 reduced by 5\%) & $0 \%$ & $\sim 0 \%$ & $20 \%$ & $31 \%$ \\
\hline
\end{tabular}

$D R+D C M$ Replace old desktops with newer machines with similar power use and migrate part of the data centre to an external data centre provider.

$T C+D C M$ Replace old desktops with thin clients and migrate part of the data centre to an external data centre provider.

Table III shows the results from the evaluation of our models for the four decision alternatives. Expected $5 y$ Energy Cost shows the impact on the goal Minimize [Energy Cost] over the period of five years with an illustration of the expected overall alternatives' cost below. Expected Scope 1\&2 Emissions shows the impact on the goal Minimize [Scope 1\&2 Emissions]. Further in the table are shown probabilities of the alternatives to reduce scope 1 and 2 emissions by 5\% from the department's current $\mathrm{CO}_{2}$ levels (first probability considers reducing ICT-related emissions only, second probability considers reducing the overall department's emissions).

\section{B. Cost-Benefit Analysis}

It is important to know whether the expected overall benefit resulting from a decision outweighs the expected overall cost and by how much. A method commonly used for such comparison is the Cost-Benefit Analysis (CBA). It assumes that a model with cost and benefit functions exists which relates various levels of goal attainment to financial interests of the stakeholders [35]. In our method, the cost benefit model can be defined in terms of the strategic goals that either directly represent the financial interests of stakeholders or can be linked to them using appropriate techniques [16], [36].

If the cost-benefit model is evaluated for inputs that have been computed using Monte Carlo simulation, one can, for each decision alternative, estimate useful measures such as the expected net benefit $(E N B)$, loss probability $(L P)$, and probable loss magnitude $(P L M)$, defined as

$$
\begin{aligned}
E N B(p) & =E[N B(p)] \\
L P(p) & =P(N B(p)<0) \\
P L M(p) & =E[N B(p) \mid N B(p)<0]
\end{aligned}
$$

Case study. We have elicited parameter values for the cost function from the Head of Technical Support Group as $90 \%$ confidence intervals. The cost of the "business as usual" scenario includes the cost of the renewal of ageing desktops. This has been estimated as a cost of $[£ 325, £ 375]$ per machine, i.e. a cost of $[£ 32,500, £ 37,500]$ for all 100 machines. The cost of the Thin Clients project is based on the cost of one thin client unit estimated at $[£ 250, £ 350]$, or $[£ 25,000, £ 35,000]$ for all 100 units.
The Data Centre Migration project involves a one-off migration cost and a yearly recurring fee for the external data management. The migration cost has been estimated to be $[£ 32,000, £ 40,000]$, with a yearly hosting fee of $[£ 25,000, £ 35,000]$.

The benefit is related to the financial savings achieved by a reduced energy consumption leading to smaller energy bills and lower CRC tax rate. The net benefit in our example is therefore defined as

$$
N B(a)=\text { Total5yExpenses }(D R)-\text { Total5yExpenses }(a)
$$

where $a$ is a decision alternative and Total5yExpenses $(D R)$ returns the expected total expenses related to the "business as usual" scenario, i.e. the renewal of the ageing desktops.

Table IV shows the results of our cost-benefit analysis. Alternative $T C+D C M$ is most profitable in terms of net benefit but has a relatively high loss probability. Second best in terms of net benefit is the alternative TC with zero probability of incurring loss.

As mentioned previously, the Technical Support Group had rejected the $D R+D C M$ and $T C+D C M$ alternatives prior to this analysis mainly due to concerns about data security. Their decision could be retrospectively interpreted in the way that the group values data security more than satisfying their overall $\mathrm{CO}_{2}$ reduction target by $31 \%$. Data security objectives could be added to the UCL strategic goal model, albeit quantifying their relationship with other sustainability goals would be difficult. Either way, our analysis provides means for exposing trade-offs among sustainability goals and other objectives, and the underlying models can be further improved based on stakeholders' feedback and reused for evaluating the impact of future project proposals.

\section{COMPUTING INFORMATION VALUE}

If the decision makers can acquire further information about the decision they face, that information may reduce their uncertainty about the impact of their decision and possibly allow them to make a better decision resulting in higher net benefit. The increase in net benefit due to the additional information is the measure of the value of this information [26]. Knowing the value of information helps decision makers prioritise which additional information they should seek - high-value information should be sought primarily, paying more for information than what it is worth should be avoided.

The expected value of total perfect information (EVTPI) is the expected gain in net benefit from having perfect information about all model parameters: 
TABLE IV. Results of the cost-benefit analysis.

\begin{tabular}{lrrrr}
\hline & \multicolumn{1}{c}{$D R$} & \multicolumn{1}{c}{$T C$} & \multicolumn{1}{c}{$D R+D C M$} & \multicolumn{1}{c}{$T C+D C M$} \\
\hline Expected 5y Energy Cost & $£ 2,238,966$ & $£ 2,203,077$ & $£ 2,040,483$ & $£ 2,004,594$ \\
Expected 5y Project Cost & $£ 35,002$ & $£ 29,983$ & $£ 215,126$ & $£ 210,108$ \\
Total 5y Expenses & $£ 2,273,968$ & $£ 2,233,061$ & $£ 2,255,609$ & $£ 2,214,701$ \\
Expected Net Benefit & $£ 0$ & $£ 40,907$ & $£ 18,359$ & $£ 59,267$ \\
Loss Probability & $0 \%$ & $0 \%$ & $44 \%$ & $30 \%$ \\
Probable Loss Magnitude & $£ 0$ & $£ 0$ & $£ 80,519$ & $£ 68,974$ \\
\hline
\end{tabular}

$$
E V T P I=E\left[\max _{a \in A} N B(a)\right]-\max _{a \in A} E[N B(a)]
$$

where $E(x)$ denotes the expectation of a random variable. With perfect information, decision makers can decide based on the true maximal net benefit for the known values of model parameters. The gain from having the perfect information is computed as the difference between the net benefit with the perfect information, i.e. $E[\max N B(a)]$, and the net benefit without the exact values, i.e. $\max E[N B(a)]$. The $E V T P I$ is always greater or equal to zero. It can be estimated from the net benefit values obtained in the previous step as

$$
E V P I=\operatorname{mean}(\operatorname{maxRow} \widehat{N B})-\max (\operatorname{meanCol} \widehat{N B})
$$

where $\widehat{N B}$ is a net benefit matrix with columns formed of the net benefit vectors computed by Monte Carlo (MC) simulation in step 3, maxRow returns a vector with the per-row maximum values of its input matrix and meanCol returns a vector with the per-column mean values of its input matrix. An illustration of computing EVTPI is shown in Table $\mathrm{V}$ for a MC simulation with only 5 scenarios (the EVTPI in our motivating example was computed for a simulation with 100,000 scenarios).

One can also compute the expected value of partial perfect information, i.e. the perfect information value of a single model parameter $\pi \in \operatorname{Par}$ ( $P$ ar is the set of all model parameters). It is defined as the gain in net benefit from having perfect information about a single model parameter $\pi$

TABLE V. Illustration of EVTPI computation from Monte Carlo simulation results. The second to fifth columns show the $\widehat{N B}$ for the four decision alternatives in 5 random scenarios. Alternative $T C+D C M$ has the highest expected net benefit in this illustration $(£ 59,487)$. Last column shows the maximum net benefit for each scenario with a mean of $£ 96,732$. We thus compute $E V T P I=£ 96,732-£ 59,487=£ 37,245$.

\begin{tabular}{rrrrrr}
\hline Scenario & $\widehat{N B}(D R)$ & $\widehat{N B}(T C)$ & $\begin{array}{l}\widehat{N B}(D R \\
+D C M)\end{array}$ & $\begin{array}{l}\widehat{N B}(T C \\
+ \text { DCM })\end{array}$ & Max \\
\hline 1 & $£ 0$ & $£ 32,980$ & $£ 70,424$ & $£ 89,000$ & $£ 89,000$ \\
2 & $£ 0$ & $£ 18,934$ & $-£ 70,140$ & $-£ 144,343$ & $£ 18,934$ \\
3 & $£ 0$ & $£ 57,463$ & $£ 42,044$ & $£ 114,345$ & $£ 114,345$ \\
4 & $£ 0$ & $£ 49,821$ & $-£ 3,100$ & $£ 207,383$ & $£ 207,383$ \\
5 & $£ 0$ & $£ 45,000$ & $£ 54,000$ & $£ 31,050$ & $£ 54,000$ \\
\hline Mean & $£ 0$ & $£ 40,840$ & $£ 18,646$ & $£ 59,487$ & $£ 96,732$ \\
\hline
\end{tabular}

$$
E V P P I(\pi)=E(\max N B(a \mid \pi))-\max E(N B(a))
$$

where $N B(a \mid \pi)$ is the net benefit given the exact values of the parameter $\pi$. Just like EVTPI, the EVPPI is always greater or equal to zero. Computing EVPPI is harder than computing EVTPI because it generally requires a computationally intensive two-level MC simulation. However, it can be substituted by an efficient, one-level simulation method that approximates the result using a segmentation approach [37].

Note that EVTPI and EVPPI computes the expected value of information about given parameters before the true values of these parameters are exposed. Once the true values of given parameters are exposed, the expected net benefit may increase or decrease. EVTPI and EVPPI thus compute how much the expected net benefit changes on average.

Case study. For our decision problem, the EVTPI is $£ 37,245$, a relatively high value. It means that eliminating all uncertainties about model parameters on average improves the expected net benefit by this amount. While in reality it will be impossible to completely eliminate the uncertainty about every single parameter, the EVTPI measure is still useful in that it gives the maximum amount that should not be exceeded to collect additional information.

The EVPPI computed for individual model parameters is shown in Table VI. We show only the top seven results because the EVPPI about the remaining parameters was negligible. Relatively high value is attached to eliminating uncertainty about the departmental server electricity use, the electricity use of the external data centre provider, the cost of an external server, and

TABLE VI. EVPPI for ICT impact model parameters.

\begin{tabular}{lr}
\hline parameter name & \multicolumn{1}{c}{ EVPPI } \\
\hline House Server Elec. Use & $£ 28,710$ \\
Ext. Server Elec. Use & $£ 12,841$ \\
Ext. Server Cost & $£ 4,739$ \\
House DCIM & $£ 3,680$ \\
External DCIM & $£ 750$ \\
Number of GPU Desktops & $£ 101$ \\
Wi-Fi Elec. Use & $£ 73$ \\
\hline
\end{tabular}


the departmental DCIM. However, collecting additional information about these parameters makes sense financially only if the cost of such additional information does not exceed the computed EVPPI values.

\section{CONCLUSION}

Implementing sustainability strategy at large organisations is an increasingly important activity. Yet the current generation of software systems supporting this activity lacks the required level of decision support. In this paper, we have presented a method for the assessment of projects' impact on key sustainability objectives under uncertainty. We believe that our application of the method to real decision alternatives at UCL provided useful insight into the impact of these alternatives on UCL's sustainability goals and helped to expose important trade-offs among sustainability goals and other objectives of the organisation.

As future work, we intend to validate our approach on a larger number of case studies at UCL and CGI. Adapting our existing sustainability goal model to different organisational context will help us abstract a set of sustainability goal templates that would facilitate the future elaboration of complex sustainability goal models at large organisations. We believe that our approach can influence the design of the next generation of professional sustainability management systems and improve their support for making complex decisions under uncertainty. In particular, computing information value will help organisations decide which measurements to improve based on the value that these measurements can bring to their decisions.

\section{ACKNOWLEDGMENT}

We thank CGI, namely Richard Copland and Mark StellaSawicki, for generously sponsoring our research. We also thank Denis Timm, the Head of Technical Support Group at the UCL Department of Computer Science, for kindly providing us with data about the department's ICT estate.
[1] IPCC Working Group, "Climate Change 2013: The Physical Science Basis,” 2013.

[2] S. Dietz and N. Stern, "Why economic analysis supports strong action on climate change: a response to the Stern Review's critics," Rev. Environ. Econ. Policy, vol. 2, no. 1, pp. 94-113, 2008.

[3] World Resources Institute, "Guide for Responsible Corporate Engagement in Climate Policy," 2013.

[4] McKinsey, "The business of sustainability," 2011.

[5] Verdantix, "Green Quadrant Sustainability Assurance 2011," 2011.

[6] N. P. Melville, "Information Systems Innovation for Environmental Sustainability," Manag. Inf. Syst. Q., vol. 34, no. 1, pp. 1-21, Mar. 2010.

[7] O. F. El-Gayar and B. D. Fritz, "Environmental menegement information systems (EMIS) for sustainable development: A conceptual overview," Commun. Assoc. Inf. Syst., vol. 17, 2006.

[8] N. P. Melville, "Environmental sustainability 2.0: empirical analysis of environmental ERP implementation," 2012.

[9] A. J. Hoffman, Carbon strategies: How leading companies are reducing their climate change footprint. University of Michigan Press, 2007.

[10] GreenBiz Group, "Six Growing Trends in Corporate Sustainability," 2012.

[11] G. Dorini, Z. Kapelan, and A. Azapagic, "Managing uncertainty in multiple-criteria decision making related to sustainability assessment," Clean Technol. Environ. Policy, vol. 13, no. 1, pp. 133-139, 2011.

[12] A. van Lamsweerde, Requirements engineering: from system goals to UML models to software specifications, vol. 13. Wiley, 2009.

[13] E. Letier and A. van Lamsweerde, "Reasoning About Partial Goal Satisfaction for Requirements and Design Engineering," SIGSOFT Softw. Eng. Notes, vol. 29, no. 6, pp. 53-62, Oct. 2004.

[14] W. Heaven and E. Letier, "Simulating and Optimising Design Decisions in Quantitative Goal Models," 2011.

[15] R. Howard, "The Foundations of Decision Analysis," IEEE Trans. Syst. Sci. Cybern., vol. 4, no. 3, pp. 211-219, 1968.

[16] D. W. Hubbard, How to Measure Anything: Finding the Value of Intangibles in Business. John Wiley \& Sons, 2010.

[17] UCL, "Carbon Management Plan.” 2011.

[18] UCL Student and Registry Services, "Student Statistics 20022013." [Online]. Available: http://www.ucl.ac.uk/srs/statistics/current.

[19] J. Cabot, S. Easterbrook, J. Horkoff, L. Lessard, S. Liaskos, and J.-N. Mazon, Integrating sustainability in decisionmaking processes: A modelling strategy. IEEE, 2009, pp. 207-210.

[20] M. Mahaux, P. Heymans, and G. Saval, "Discovering Sustainability Requirements: An Experience Report," 2010.

[21] B. Penzenstadler and H. Femmer, "A Generic Model for Sustainability with Process- and Product-specific Instances," in Proceedings of the 2013 Workshop on Green in/by Software Engineering, 2013, pp. 3-8.

[22] A. van Lamsweerde, Requirements Engineering. 2009.

[23] J. Lung, "Inflo Workbench," 2011. [Online]. Available: https://inflo.cs.toronto.edu. [Accessed: 13-Aug-2011].

[24] J. Lung and S. Easterbrook, "Inflo: Collaborative Reasoning via Open Calculation Graphs," in Proceedings of the ACM 2012 Conference on Computer Supported Cooperative Work, 2012, pp. 1199-1202. 
[25] E. Letier, D. Stefan, and E. T. Barr, "Uncertainty, Risk, and Information Value in Software Requirements and Architecture," in ICSE2014, 2014.

[26] R. A. Howard, "Information Value Theory," Syst. Sci. Cybern. IEEE Trans., vol. 2, no. 1, pp. 22-26, Aug. 1966.

[27] D. Stefan, E. Letier, M. Barrett, and M. Stella-Sawicki, "Goal-oriented System Modelling for Sustainability Management Systems,” 2011.

[28] Green UCL, "UCL Sustainability Strategy," 2012.

[29] DEFRA, "Guidance on how to measure and report your greenhouse gas emissions," 2009.

[30] HEFCE, "Carbon reduction target and strategy for higher education in England," 2010.

[31] Environment Agency, "CRC Energy Efficiency Scheme," 2012. .

[32] UK Government, "SusteIT ICT energy and carbon footprinting tool," 2013. [Online]. Available: https://www.gov.uk/government/publications/susteit-ictenergy-and-carbon-footprinting-tool.

[33] A. O'Hagan, Uncertain Judgements: Eliciting Experts' Probabilities. John Wiley \& Sons, 2006.

[34] Carbon Trust, "Conversion factors," 2013.

[35] A. E. Boardman, "Cost-benefit analysis," 2010.

[36] G. Adzic, Impact Mapping: Making a big impact with software products and projects. Provoking Thoughts, 2012.

[37] M. Sadatsafavi, N. Bansback, Z. Zafari, M. Najafzadeh, and C. Marra, "Need for speed: An efficient algorithm for calculation of single-parameter expected value of partial perfect information," Value Heal., vol. 16, no. 2, pp. 438-448, 2013. 